\title{
Effective Personalized Treatment of Advanced Hepatic Carcinoma based on Sorafenib
}

\author{
Wang Qun*, Yin Tao, Duan Chuan Yi, Lu Weijun, Wang Yang Fu and Chang Bo
}

Department of General Surgery, Zhongnan Hospital of Wuhan University, Hubei Province, PR China

\begin{abstract}
Background: Hepatic carcinoma (HCC) has rised in China because of hepatitis B virus(HBV) effection, it has been famous for devastating malignancy as well as little treatment effect for HCC Sorafenib, has been successfully applied for solid tumors such as renal cancer, hepatocellular carcinoma. Sorafenib used alone or combination with others can induce growth-inhibition and apoptosis in vitro experiment. Sorafenib now was suggested to advanced hepatic carcinoma patient unqualitied for hepatectomy and transplant.
\end{abstract}

Methods: A team of advanced hepatic carcinoma patients were enrolled for Sorafenib monotherapy or combination with HIAC,TACE, systemtic chemotherapy with arsenic trioxide and octreotide on base of personal disease progress CT and level of AFP were used to assess the tumor effect for decision of next treatment plan, An individualization treatment plan was performed ultimately.

Result: Patients of initially HCC were extended survival time by Sorafenib monotherapy or combination with HIAC and TACE. TACE made hepatic lesion stable, added therapy of arsenic trioxide could be able to make TACErefractory and lung metatasis lesion under control.

Conclusion: Sorafenib monotherapy or combination with others added therapy on needs of patients personal disease progress can extend survival time. Sorafenib applied individually may be more effective.

Keywords: Personalized treatment; Advanced hepatic carcinoma; Sorafenib

\section{Case Reports}

\section{Patients' collections}

A team of advanced hepatic carcinoma patients including: initially unresectable hepatic carcinoma, extrahepatic metastasis after radical surgical resection, recurrences inside liver only after operation were enrolled who survival time were all over 2 years [1,2]. Thirteen patients were enrolled, two women and eleven men included, two patients suffered from intrahepatic cholangiocarcinoma were confirmed by biopsy-proven one was treated with Sorafenib monotherapy, another was administered with Sorafenib and hepatic HIAC reported by us before $[3,4]$. The left eleven patients were confirmed to be hepatocelluar carcinoma. Twelve of them were infected with hepatitis B virus. Three of 13 were demonstrated with portal vein tumor thrombosis by dynamic contrast enhanced CT, one suffered from portal vein tumor thrombosis in portal trunk and both first branches as well as oesophageal variceal bleeding. Three of 13 belong to extrahepatic metastasis two was lungs; one was both lungs and bones (Table 1).

\section{Treatment plan decision}

Personal treatment scheme was made according to different personal disease progress of sufferers as follows : 6 cases initially unrespectable, 3 of them sorafenib was administered alone, one of them combination with octreotide on account of bleeding from portal vein tumor thrombus and hepatic cirrhosis, one intrahepatic cholangiocarcinoma (ICC) combination with hepatic arterial infusion chemotherapy of 5-Fu/LV + oxaliplatin + hydroxycamptothecine, another intrahepatic cholangiocarcinoma was administered sorafenib monotherapy. 4 cases of intrahepatic recurrences after radical surgical resection all of them alpha fetoprotein (AFP) downsized in two months after operation, one accepted Arsenic trioxide as $10 \mathrm{mg} / \mathrm{d}$ intravenously in total $2 \mathrm{mg} / \mathrm{kg}$ for reason of alpha fetoprotein (AFP) upraising and
TACE-refractory, Three without enough future remnant liver for resection accepted Sorafenib combination with TACE. In hepatic recurrences were treated with transhepatic arterial chemotherapy and TACE who had no chance of resection, For the left 3 extrahepatic metastasis patients, one with both lungs and bone metastasis and two with lungs metastasis, Arsenic trioxide(AT) was used as systemic chemotherapy which was applied $10 \mathrm{mg} / \mathrm{d}$ intravenously accumulation to $1 \mathrm{mg} / \mathrm{kg}$, Sorafenib was suggested as routine, Sorafenib was applied firstly as $400 \mathrm{mg}$ twice a day. There were no dose-limiting toxicities found who received $400 \mathrm{mg}$ twice a day. Combined with TACE, some patients suffered from skin hyperkeratosis and vomit then it was used as $200 \mathrm{mg}$ bid, Efficacy of treatment was evaluated with dynamic

\begin{tabular}{|c|c|c|}
\hline $\begin{array}{c}\text { Initially } \\
\text { unresectable }\end{array}$ & Extrahepatic metatasis & Recurrences inhepatic \\
\hline $\begin{array}{c}\text { Sorafenib }(\mathrm{HCC})+ \\
\text { TACE }\end{array}$ & $\begin{array}{c}\text { Sorafenib }+ \text { TACE }+ \text { AT (bones } \\
\text { lungs) }\end{array}$ & Sorafenib + TACE \\
\hline Sorafenib (HCC) & Sorafenib + TACE + AT (lungs) & Sorafenib + TACE + AT \\
\hline Sorafenib (HCC) & Sorafenib + TACE + AT (lungs) & Sorafenib + TACE \\
\hline Sorafenib + octreotide & & Sorafenib + AT \\
\hline $\begin{array}{c}\text { Sorafenib + } \\
\text { infusion(ICC) }\end{array}$ & & \\
\hline Sorafenib (ICC) & & \\
\hline
\end{tabular}

Table 1: All of the thirteen patients personalized treatment plan.

*Corresponding author: Wang Qun, Department of General Surgery, Zhongnan Hospital of Wuhan University, Hubei Province, PR China, Tel: +86-15926270216; E-mail: swander@126.com

Received January 09, 2016; Accepted May 02, 2016; Published May 06, 2016

Citation: Qun W, Tao Y, Yi DC, Weijun L, Fu WY, et al. (2016) Effective Personalized Treatment of Advanced Hepatic Carcinoma based on Sorafenib. J Integr Oncol 5: 164. doi:10.4172/2329-6771.1000164

Copyright: (C) 2016 Qun W, et al. This is an open-access article distributed unde the terms of the Creative Commons Attribution License, which permits unrestricted use, distribution, and reproduction in any medium, provided the original author and source are credited. 
contrast enhanced computed tomography (CT) scan. In brief, for extrahepatic metastasis of HCC patients Sorafenib combination with Arsenic trioxide systemic therapy may be useful. TACE was added for hepatic lesion, Recurrences inhepatic patients accepted for Sorafenib as well as TACE, Arsenic trioxide could be administered to patients as rescue therapy as soon as TACE-refractory was confirmed.

\section{CT evaluation}

All candidates were followed up bimonthly; daymaic contrast enhanced computed tomography scans were performed to evaluate progress of lesion in liver. Lungs were all scanned for metastasis; size and count of hepatic lesions were compared with before, Lymph nodes greater than $2 \mathrm{~cm}$ could be regarded as malignant involvement. The efficacy of TACE was assessed by performing dynamic computed tomography before and 4 weeks after the TACE treatment, plain CT was applied to assess the uptake of Lipiodol. The rate of reduction of the necrotic area was estimated from the Lipiodol accumulation observed on CT after TACE. The tumor-necrotizing effect and tumor size reduction rate were assessed on the basis of the reduction in tumor size or the disappearance of hypervascularity from the nodule using bi-dimensional measurements as mentioned by Yamanaka et al.[5]. TACE-refractory was considered if no or little Lipiodol was demonstrated on CT. As far as hepatocellular carcinoma with portal vein thrombosis, size reduction and (or) revascularization of portal vein tumor thrombosis displayed on CT were considered effective treatment by Sorafenib Decreased hepatic lesions in size or numbers with stable tumor thrombosis were also considered effective.

\section{Follow up}

All patients followed up monthly for decision of plan in next step, daymaic contrast enhanced computed tomography were asked for bimonthly, Results of laboratory examination such as CA199 CEA AFP, liver function blood cell count and thrombin time test were necessary for monthly Sorafenib was administered for $400 \mathrm{mg}$ twice a day as routine, Adverse events of Sorafenib such as diarrhea hand-foot skin reaction were reported as a common cause for drug discontinuation referred to dispensatory. Dose reduction of sorafenib (200 mg bid) was allowed for drug-related toxicities. Oesophageal varices bleeding was considered to be a sign of drug discontinuance as soon as bleeding was made sure controlled drug continuance begin as dose of $200 \mathrm{mg}$ per day followed by $200 \mathrm{mg}$ twice a day and then 400 mg twice a day ultimately 6 patients including 3 caused by diarrhea and 3 result from hand-foot skin had to reduce to $200 \mathrm{mg}$ twice a day to release such adverse events One person had a temporary interruption for reason of oesophageal varices bleeding till it was controlled by octreotide. The left 7 patients including 2 ICC patients suffered no fierce adverse event besides weight reduction. Results of laboratory examination of CA19-9 CEA AFP were collected to be compared with before. Blood cell count and liver function were made necessary for next plan. Child C was forbidden to accept Sorafenib, for two patients percutaneous partial splenic artery interventional remobilization was performed respectively as result of the blood count of platelet of patient decreased to below $5 \times 10^{9}$ Daymaic contrast enhanced and plain CT were performed to demonstrate progress of hepatic and lung lesions The efficacy of TACE was also evaluated by CT.

\section{Discussion}

There is no regular treatment strategy for advanced hepatic carcinoma for it was difficult to be controlled Patients with unresectable or metastatic HCC have median survival of a few months hepatocellular carcinoma was generally considered to be highly resistant to conventional chemotherapeutic agents administered either alone or in combination [6]. Chemotherapy for advanced HCC has limited response rates about $15-25 \%$ and provides a marginal survival benefit [7] all the patients enrolled by us had a over two years survival time with personalized treatment based on Sorafenib. Sorafenib is the only taken oral chemotherapeutic agents for advanced HCC. There are many strategies reported about Sorafenib, as a result it was still controversial that Sorafenib should be applied alone or combination with others, For example Sorafenib combination TACE was strongly suggested by many researchers $[8,9]$. On the other hand the combination was confirmed to be unuseful for some TACErefractory patients. As far as we know Sorafenib used as monotherapy or combination should be strongly dependent on personal response and different stage of hepatocellular carcinoma. We apply Sorafenib to intrahepatic cholangiocarcinoma based on at least two mechanisms account for the observed effects: Sorafenib's ability to arrest the cell cycle in the G1/G0 phase as well as inducing inducing apoptosis [10]. Two of our patients accepted monotherapy and combination with HIAC respectively. Both of them had good response but for definite mechanism needed to be figured out. For the combination therapy we speculated that down-regulation expression of Mcl-1 by Sorafenib made effect which had been implicated in resistance to anticancer drug [4]. Now it was also discovered by us that monotherapy of Sorafenib was feasible for intrahepatic cholangiocarcinoma. It is concluded by us that Sorafenib may also play important role in treatment of intrahepatic cholangiocarcinoma (Table 2).

As to the patient of hepatocellular carcinoma with portal vein tumor thrombosis and oesophageal variceal bleeding. Octreotide was added to control bleeding but both portal vein tumor thrombosis and hepatic lesion were decreased simultaneously by Sorafenib it was concluded by us that control of oesophageal variceal must be based on effective treatment of portal vein tumor thrombosis and hepatocellular carcinoma simultaneously because it was argued that hepatocellular carcinoma disease control was the most significant and independent factor for aggravation of oesophageal varices [11]. As a result, we believe that Sorafenib is qualified for hepatocellular carcinoma with portal vein tumor thrombosis if oesophageal variceal bleeding is made under control. Sorafenib monotherapy makes effect in three of our candidates including one with portal vein tumor thrombosis in the portal trunk; both hepatic lesions and tumor thrombosis were decreased displayed on daymaic contrast enhanced and plain CT initially (Figure 1). Therefore no added therapy was necessary for them. Three of extrahepatic metastasis patients were made stable with Sorafenib combination arsenic trioxide the reason that they accepted combination therapy because it was demonstrated that the presence of lung metastasis predicted poor response to Sorafenib in advanced HCC patients [12] and arsenic trioxide has been found to have significant antitumor metastasis effect in hepatocellular carcinoma [13,14]. It has been reported in China that Sorafenib combination arsenic trioxide were more effective inducing hepatocellular carcinoma cells into apoptosis in vitro experiment [15]. Arsenic trioxide was discovered to induce G2/M arrest in hepatocellular carcinoma cells by increasing the tumor suppressor PTEN expression [16]. Arsenic trioxide was

\begin{tabular}{|l|c|c|c|c|c|c|}
\hline & \multirow{2}{*}{ No treatment } & \multicolumn{5}{|c|}{ Treatment with Sorafenib } \\
\cline { 3 - 7 } & & $\mathbf{2}$ months & 4 months & $\mathbf{6}$ months & $\mathbf{1 2}$ months & 20 months \\
\hline AFP & $(\mathrm{ng} / \mathrm{ml}) 6906$ & 361 & 3.8 & 1.0 & 1.8 & 2.0 \\
\hline Size & $(\mathrm{cm}) \quad 7.5 \times 5.9$ & $3.0 \times 2.5$ & $2.5 \times 2.0$ & $2.3 \times 1.9$ & $2.1 \times 2.0$ & $2.5 \times 2.1$ \\
\hline
\end{tabular}

Table 2: Level of AFP and size of tumor before and after chemotherapy. 
Citation: Qun W, Tao Y, Yi DC, Weijun L, Fu WY, et al. (2016) Effective Personalized Treatment of Advanced Hepatic Carcinoma based on Sorafenib. J Integr Oncol 5: 164. doi:10.4172/2329-6771.1000164

Page 3 of 3
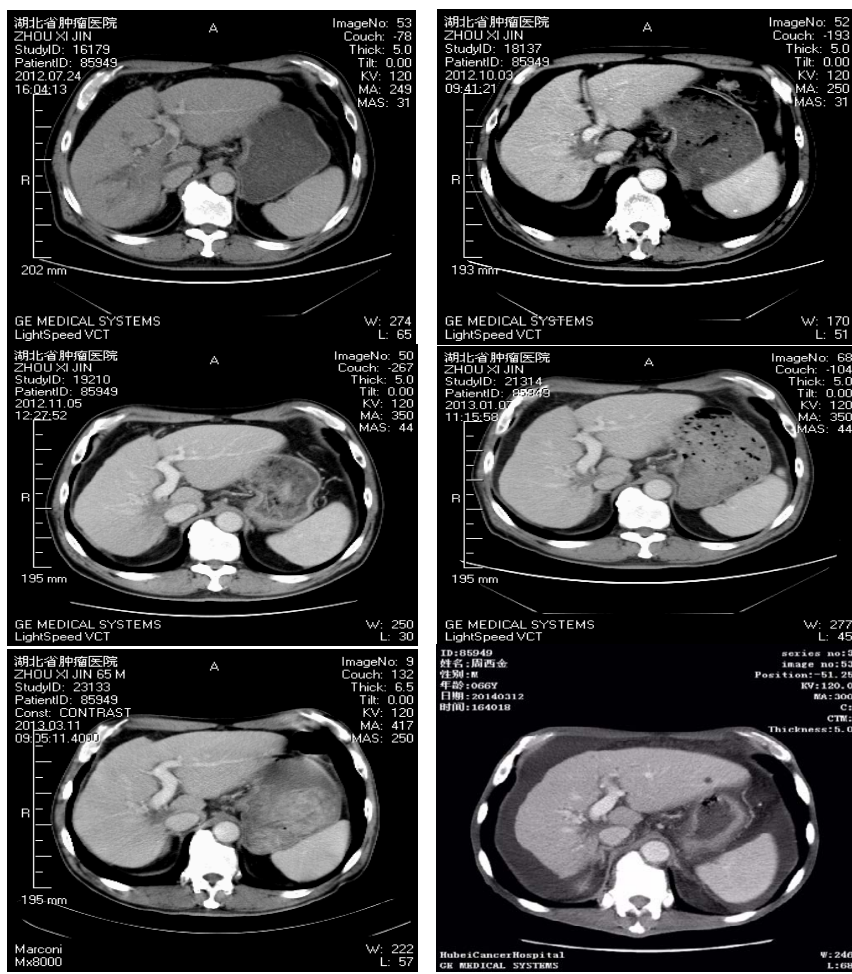

Figure 1: In the pictures a patient was discovered liver cancer with portal vein tumor thrombosis in the portal trunk and both first branches of the portal vein, after 3 month of therapy with Sorafenib monotherapy 400mg Bid portal vein tumor thrombosis was succumbed, and then hepatic low-density lesion was decreased and made stable. AFP continued in low level.

also effective in treating multidrug resistant human hepatocellular carcinoma cells over expressing P-glycoprotein and exerted its effect via induction of apoptosis and. It is speculated that Arsenic trioxide release human hepatocellular carcinoma cells multidrug resistant to Sorafenib. It was discovered that sorafenib induced Mcl-1 down-regulation might sensitize cells to other agents that trigger apoptosis through the mitochondrial pathway [17]. Sorafenib added TACE were applied to 3 patients of inhepatic recurrences who had good response to TACE All the patients were evaluated by CT to decide personal treatment plan. Once TACE-refractory or size expanded was demonstrated on CT, Sorafenib combination arsenic trioxide was used systematically.

In China the current cost of the drug precludes sorafenib from becoming a commonly used treatment for advanced hepatocellular carcinoma. Sorafenib could be regarded as means to save the situation besides for hepatectomy, transcatheter arterial embolization (TAE), percutaneous ethanol injection (PEI), microwave coagulation therapy (MCT) and radiofrequency ablation (RFA). We only provide 13 patients for share. It maybe not enough to be confirmed but for the first time we create the personalized treatment plan based on need of every patient's disease progress what we need to do is to expand the plan.

\section{References}

1. Llovet JM, Ricci S, Mazzaferro V, Hilgard P, Gane E, et al. (2008) Sorafenib in advanced hepatocellular carcinoma. N Engl J Med 359: 378-390.

2. Colombo M (2009) Sorafenib in advanced hepatocellular carcinoma: a further step toward personalized therapy of liver cancer. Gastroenterology 136: 1832 1835.

3. Huether A, Hopfner M, Baradari V, Schuppan D, Scherübl H (2007) Sorafenib alone or as combination therapy for growth control of cholangiocarcinoma. Biochem Pharmacol 73: 1308-1317.

4. Qun W, Tao Y (2010) Effective Treatment of Advanced Cholangiocarcinoma by Hepatic Arterial Infusion Chemotherapy Combination with Sorafenib: One Case Report from China. Hepato-Gastroenterology 57: 426-429.

5. Yamanaka K, Hatano E, Kitamura K, lida T, Ishii T, et al. (2012) Early evaluation of transcatheter arterial chemoembolization-refractory hepatocellular carcinoma. J Gastroenterol 47: 343-346.

6. Llovet JM, Burroughs A, Bruix J (2003) Hepatocellular carcinoma. Lancet 362 1907-1917.

7. Han KH, Park JY (2008) Chemotherapy for advanced hepatocellular carcinoma Journal of Gastroenterology and Hepatology 23: 682-684.

8. Furuse $\mathrm{J}$ (2008) Sorafenib for the treatment of unresectable hepatocellula carcinoma. See comment in PubMed Commons below Biologics 2: 779-788.

9. Dufour JF, Hoppe H, Heim MH, Helbling B, Maurhofer O, et al. (2010) Continuous administration of sorafenib in combination with transarterial chemoembolization in patients with hepatocellular carcinoma: results of a phase I study. Oncologist 15: 1198-1204.

10. Huether A, Höpfner M, Baradari V, Schuppan D, Scherübl H (2007) Sorafenib alone or as combination therapy for growth control of cholangiocarcinoma. Biochem Pharmacol 73: 1308-1317.

11. Kodama H, Aikata H, Murakami E, Miyaki D, Nagaoki Y, et al. (2011) Clinica outcome of esophageal varices after hepaticarterial infusion chemotherapy for advanced hepatocellular carcinoma with major portal vein tumor thrombus. Hepatology Research 41: 1046-1056.

12. Yau T, Chan P, Ng KK, Chok SH, Cheung TT, et al. (2009) Phase 2 open-labe study of single-agent sorafenib in treating advanced hepatocellular carcinoma in a hepatitis B-endemic Asian population: presence of lung metastasis predicts poor response. Cancer 115: 428-436.

13. Shi Y, Xia J, Yang TH (2011) Inhibitory effect of arsenic trioxide on the pulmonary metastasis of melanoma B16 cells.

14. Zhao XS, Song PL, Sun B, Jiang HC, Liu TF (2009) Arsenic trioxide inhibits metastatic potential of mouse hepatoma $\mathrm{H} 22$ cells in vitro and in vivo. Hepatobiliary Pancreat Dis Int 8: 510-517.

15. Wu J, Luo RC, Zhang H, Cui YZ (2008) Inhibitory effect of sorafenib combined with arsenic trioxide on hepatocellular carcinoma cells. Nan Fang Yi Ke Da Xue Xue Bao 28: 639-641.

16. Zhang $X$, Jia S, Yang S, Yang Y, Yang T, et al. (2012) Arsenic trioxide induces G2/M arrest in hepatocellular carcinoma cells by increasing the tumor suppressor PTEN expression. J Cell Biochem 113: 3528-3535.

17. Yu C, Bruzek LM, Meng XW, Gores GJ, Carter CA, et al. (2005) The role of $\mathrm{Mcl}-1$ downregulation in the proapoptotic activity of the multikinase inhibitor BAY 43-9006. Oncogene 24: 6861-6869. 